ANNALES

POLONICI MATHEMATICI

LXXVIII.1 (2002)

\title{
Width asymptotics for a pair of Reinhardt domains
}

\author{
by A. Aytuna (Ankara), A. Rashkovskin (Kharkov) and \\ V. ZAhariuta (Rostov-na-Donu and Ankara)
}

Abstract. For complete Reinhardt pairs "compact set - domain" $K \subset D$ in $\mathbb{C}^{n}$, we prove Zahariuta's conjecture about the exact asymptotics

$$
\ln d_{s}\left(A_{K}^{D}\right) \sim-\left(\frac{n ! s}{\tau(K, D)}\right)^{1 / n}, \quad s \rightarrow \infty,
$$

for the Kolmogorov widths $d_{s}\left(A_{K}^{D}\right)$ of the compact set in $C(K)$ consisting of all analytic functions in $D$ with moduli not exceeding 1 in $D, \tau(K, D)$ being the condenser pluricapacity of $K$ with respect to $D$.

1. Introduction. The Kolmogorov widths of a compact set $A$ in a Banach space $X$ are the numbers

$$
d_{s}(A)=d_{s}(A, X):=\inf _{L} \sup _{x \in A} \inf \left\{\|x-y\|_{X}: y \in L\right\}, \quad s \in \mathbb{Z}_{+},
$$

where $L$ runs through the set of all $s$-dimensional subspaces of $X$.

Let $K$ be a compact subset of an open set $D \subset \mathbb{C}^{n}$ and $A_{K}^{D}$ be the subset of $C(K)$ consisting of all analytic functions in $D$ whose moduli do not exceed 1 in $D$. For quite general pairs $(K, D)$, the weak asymptotics

$$
\ln d_{s}\left(A_{K}^{D}\right) \asymp s^{1 / n}, \quad s \rightarrow \infty,
$$

is known to be true; it is equivalent to the result of Kolmogorov [8] on the asymptotics for the $\varepsilon$-entropy of the set $A_{K}^{D}$ :

$$
H_{\varepsilon}\left(A_{K}^{D}\right) \asymp\left(\ln \frac{1}{\varepsilon}\right)^{n+1}, \quad \varepsilon \rightarrow 0
$$

2000 Mathematics Subject Classification: 32A07, 32U20.

Key words and phrases: Kolmogorov width, pluricapacity, Reinhardt domain.

The second author was partially supported by NATO-TÜBITTAK Fellowship Program and INTAS 99-00089 grant, and the third author by NATO-TÜBİTAK D-Program. 
(the equivalence of the asymptotics (1) and (2) follows from the results of Mityagin [10] and Levin-Tikhomirov [9]). Kolmogorov also suggested the conjecture that in the one-dimensional case, for quite general pairs $(K, D)$, the strong asymptotics

$$
\ln d_{s}\left(A_{K}^{D}\right) \sim-\frac{s}{\tau(K, D)}
$$

should be true, where $\tau(K, D)$ is the Green capacity of $K$ with respect to $D$ (or the capacity of the condenser $(K, D)$, see e.g. [7]). This conjecture was confirmed by many authors $([5,3,17,11,6,16,14]$; for more details see [19]).

The problem of existence of the strong asymptotics

$$
\ln d_{s}\left(A_{K}^{D}\right) \sim-\sigma s^{1 / n}
$$

for several variables was considered in [19], where some estimates from above and from below for the numbers $d_{s}\left(A_{K}^{D}\right)$ were obtained and, as a consequence, some sufficient conditions for the existence of the asymptotics (4) were presented. Under those conditions (they seem to be quite general, although it is not clear yet how to check them even for relatively simple specific pairs $(K, D))$, the constant $\sigma$ has a natural expression:

$$
\sigma=\left(\frac{n !}{\tau(K, D)}\right)^{1 / n},
$$

where $\tau(K, D)=(2 \pi)^{-n} C(K, D)$ and $C(K, D)$ is the pluricapacity of $K$ with respect to $D$, introduced by Bedford and Taylor [4]. On the ground of this result it was conjectured ([19], Conjecture 3.1.3) that this asymptotics should be true under quite general assumptions about the pairs $(K, D)$.

Here we prove this conjecture for any pair $K=\bar{D}_{0}, D=D_{1}$, where $D_{\nu}, \nu=0,1$, are bounded complete logarithmically convex Reinhardt (i.e. $n$-circular) domains (Theorem 5). The main steps of the proof are as follows. First, the strong asymptotics (4) is valid with some constant $\sigma$ expressed through the support functions of the domains $D_{\nu}$ (see Section 3), which was proved independently by L. Ronkin and V. Zahariuta (unpublished). On the other hand, the pluripotential $\omega\left(D_{1}, \bar{D}_{0} ; z\right)$ can also be represented by means of the support functions [19]. To compute the pluricapacity, we reduce the problem to the real Monge-Ampère operator for convex functions, which can be expressed in geometric terms [13]. The calculation gives us exactly the value $\sigma$ obtained.

2. Preliminaries. Let $\Omega$ be a bounded pseudoconvex domain in $\mathbb{C}^{n}$ and $K$ be a compact subset of $\Omega$. The Green pluripotential $\omega(\Omega, K ; z)$ of $K$ 
with respect to $\Omega$ is defined as

$$
\omega(\Omega, K ; z)=\varlimsup_{\zeta \rightarrow z} \sup \left\{u(\zeta): u \in \operatorname{PSH}(\Omega), u \leq 1,\left.u\right|_{K} \leq 0\right\},
$$

$\operatorname{PSH}(\Omega)$ being the cone of all plurisubharmonic functions in $\Omega$.

The image $\left(d d^{c} u\right)^{n}$ of any bounded plurisubharmonic function $u$ in $\Omega$ under the complex Monge-Ampère operator (see [4]) is a non-negative Borel measure on $\Omega$ (here $d=\partial+\bar{\partial}, d^{c}=i(\bar{\partial}-\partial)$ ). The pluricapacity $\tau(K, \Omega)$ of $K$ with respect to $\Omega$ (in other words, of the condenser $(K, \Omega)$ ) is the value

$$
\tau(K, \Omega)=(2 \pi)^{-n} \int_{K}\left(d d^{c} \omega(\Omega, K ; z)\right)^{n},
$$

which differs from the Bedford-Taylor pluricapacity ([4]) only by a constant factor.

Given a bounded complete logarithmically convex Reinhardt domain $D \subset \mathbb{C}^{n}$, its support function is

$$
h_{D}(\theta)=\sup \left\{\sum \theta_{k} \log \left|z_{k}\right|: z \in D\right\}, \quad \theta \in \mathbb{R}_{+}^{n},
$$

where $\mathbb{R}_{+}^{n}=\left\{\theta=\left(\theta_{\nu}\right) \in \mathbb{R}^{n}: \theta_{\nu} \geq 0, \nu=1, \ldots, n\right\}$. It is a convex homogeneous function in $\mathbb{R}_{+}^{n}$ such that

$$
D=\left\{z \in \mathbb{C}^{n}: \sum \theta_{k} \log \left|z_{k}\right| \leq h_{D}(\theta), \theta \in \Sigma\right\},
$$

where $\Sigma:=\left\{\theta=\left(\theta_{1}, \ldots, \theta_{n}\right) \in \mathbb{R}_{+}^{n}: \sum_{k=1}^{n} \theta_{k}=1\right\}$.

Let $D_{\nu}, \nu=1,2$, be a pair of bounded complete logarithmically convex Reinhardt domains, and $\bar{D}_{0} \subset D_{1}$. The following formula for the pluripotential of $\bar{D}_{0}$ with respect to $D_{1}$ was given in [19], Proposition 1.4.3:

$$
\omega\left(D_{1}, \bar{D}_{0} ; z\right)=\sup \left\{\frac{\sum \theta_{k} \log \left|z_{k}\right|-h_{D_{0}}(\theta)}{h_{D_{1}}(\theta)-h_{D_{0}}(\theta)}: \theta \in \Sigma\right\}
$$

for $z \in D_{1} \backslash \bar{D}_{0}$, and $\omega\left(D_{1}, \bar{D}_{0} ; z\right)=0$ for all $z \in \bar{D}_{0}$ (see Lemmas 1 and 4 below). Note that, due to homogeneity of the support functions, the set $\Sigma$ can be changed to $\mathbb{R}_{+}^{n}$ in (7) and (8).

3. Width asymptotics. First we consider a Hilbert version of the problem about the asymptotics (4), which is much more convenient to study. Let $D_{\nu}, \nu=0,1$, be bounded complete logarithmically convex Reinhardt domains such that $\bar{D}_{0} \subset D_{1}$ and $H_{\nu}$ be any pair of Hilbert spaces such that there are linear continuous embeddings

$$
A\left(\bar{D}_{1}\right) \subset H_{1} \subset A\left(D_{1}\right) \subset A\left(\bar{D}_{0}\right) \subset H_{0} \subset A\left(D_{0}\right) .
$$

Consider a common orthogonal basis $\left\{e_{j}(z)\right\}_{i \in \mathbb{N}}$ for the spaces $H_{0}$ and $H_{1}$, which we suppose being normalized and rearranged in such a way that

$$
\left\|e_{j}\right\|_{H_{0}}=1, \quad \mu_{j}\left(H_{0}, H_{1}\right):=\left\|e_{j}\right\|_{H_{1}} \uparrow \infty .
$$


The following fact is a particular case of well known results ([19], 3.1.2, see also $[18,1])$.

Lemma 1. Let $H_{0}, H_{1}$ be any pair of Hilbert spaces complying with the linear continuous embeddings (9) and $\left\{e_{j}(z)\right\}$ be their common orthogonal basis satisfying the conditions (10). Then

$$
\omega\left(D_{1}, \bar{D}_{0} ; z\right)=\limsup _{\zeta \rightarrow z} \limsup _{j \rightarrow \infty} \frac{\ln \left|e_{j}(\zeta)\right|}{\ln \mu_{j}\left(H_{0}, H_{1}\right)}
$$

for $z \in D_{1} \backslash \bar{D}_{0}$. Therefore we have the asymptotics

$$
\ln \mu_{j}\left(H_{0}, H_{1}\right) \sim-\ln d_{j}\left(A_{D_{1}}^{\bar{D}_{0}}\right), \quad j \rightarrow \infty .
$$

Thus the problem about the asymptotics (4), in the case $K=\bar{D}_{0}, D=D_{1}$, is reduced to the problem about the asymptotics

$$
\ln \mu_{j}\left(H_{0}, H_{1}\right) \sim \sigma j^{1 / n}, \quad j \rightarrow \infty,
$$

in view of the fact that neither the existence of such asymptotics nor the constant $\sigma$ depends on the concrete choice of the spaces $H_{0}, H_{1}$ ([19], 3.1.2). Therefore we choose our Hilbert spaces in a way most convenient for calculations, so that the system of all monomials $g_{j}(z)=z^{k(j)}$ enumerated according to non-decreasing degrees $s(j):=k_{1}(j)+\ldots+k_{n}(j) \uparrow \infty$ forms a common orthogonal basis for $H_{0}, H_{1}$. Namely, we set $(\nu=0,1)$

$$
H_{\nu}:=\left\{x=\sum_{j=1}^{\infty} c_{j} g_{j}:\|x\|_{H_{\nu}}:=\left(\sum_{j=1}^{\infty}\left|c_{j}\right|^{2} \exp 2 h_{D_{\nu}}(k(j))\right)^{1 / 2}<\infty\right\} .
$$

It can be easily checked that the Hilbert spaces (13) satisfy the conditions of Lemma 1 and, by construction, we have

$$
\ln \mu_{j}\left(H_{0}, H_{1}\right)=h_{D_{1}}(k(j))-h_{D_{0}}(k(j)), \quad j \in \mathbb{N} .
$$

Instead of directly studying the asymptotics of this sequence it is more convenient to consider its counting function

$$
\varphi(t):=\left|\left\{j: \ln \mu_{j}\left(H_{0}, H_{1}\right) \leq t\right\}\right|=\left|\left\{k \in \mathbb{Z}_{+}^{n}: h_{D_{1}}(k)-h_{D_{0}}(k) \leq t\right\}\right|,
$$

where $|A|$ denotes the number of elements of a finite set $A$. Then to prove the asymptotics (12) it is sufficient to show that (see, e.g., [2])

$$
\varphi(t) \sim(t / \sigma)^{n}, \quad t \rightarrow \infty .
$$

The value $\varphi(t)$ is just the number of points $k \in \mathbb{Z}_{+}^{n}$ lying in the closed domain $t \Theta, t>0$, where

$$
\Theta=\left\{\theta \in \mathbb{R}_{+}^{n}: h_{D_{1}}(\theta)-h_{D_{0}}(\theta) \leq 1\right\} .
$$

Now we use the following elementary fact (see, e.g., [15]). 
Lemma 2. Let $G$ be a closed domain in $\mathbb{R}^{n}$ measurable in the sense of Jordan and $\varphi(t):=\left|\left\{k \in \mathbb{Z}^{n}: k \in t \bar{G}\right\}\right|$. Then

$$
\varphi(t) \sim t^{n} \operatorname{Vol} G, \quad t \rightarrow \infty .
$$

Since the domain (15) is obviously measurable in the sense of Jordan, the following theorem is proved.

TheOREM 3. For K,D satisfying the condition of this section, the asymptotics (4), (12) hold with the constant

$$
\sigma=\left(\frac{1}{\operatorname{Vol} \Theta}\right)^{1 / n} .
$$

From (11), due to the special choice of Hilbert spaces, we can also obtain

Lemma 4. The formula (8) is true.

Since the proof is only sketched in [19], here we consider it in more detail. Applying (11) to the basis $e_{j}(z)=\exp \left\{-h_{D_{0}}(k(j))\right\} z^{k(j)}, j \in \mathbb{N}$, and taking into account (14), we get

$$
\omega\left(D_{1}, \bar{D}_{0} ; z\right)=\limsup _{\zeta \rightarrow z} \limsup _{j \rightarrow \infty} \frac{\sum_{s=1}^{n} k_{s}(j) \ln \left|z_{s}\right|-h_{D_{0}}(k(j))}{h_{D_{1}}(k(j))-h_{D_{0}}(k(j))} .
$$

Setting $\theta(j)=\left(\theta_{s}(j)\right):=k(j) /|k(j)|$ and using the homogeneity of the support functions, we can rewrite the right-hand side of this equality in the form (the first upper limit can be dropped, because the expression within it turns out to be continuous)

$$
\sup _{\theta \in \Sigma} \limsup _{\theta(j) \rightarrow \theta} \frac{\sum_{s=1}^{n} \theta_{s}(j) \ln \left|z_{s}\right|-h_{D_{0}}(\theta(j))}{h_{D_{1}}(\theta(j))-h_{D_{0}}(\theta(j))}=\sup _{\theta \in \Sigma} \frac{\sum_{s=1}^{n} \theta_{s} \ln \left|z_{s}\right|-h_{D_{0}}(\theta)}{h_{D_{1}}(\theta)-h_{D_{0}}(\theta)}
$$

which completes the proof of the lemma.

In the next section we will compute the pluricapacity of the condenser $\left(\bar{D}_{0}, D_{1}\right)$; then the following main result will be derived immediately from Theorem 3.

Theorem 5. For any pair $(K, D)$ with $K=\bar{D}_{0} \subset D_{1}=D$, were $D_{\nu}$ are bounded complete logarithmically convex Reinhardt domains, the asymptotics (4) is true with the constant (5).

4. Pluricapacity of a pair of Reinhardt domains. In what follows we will employ the correspondence between multicircular domains in $\mathbb{C}^{n}$ and convex ones in $\mathbb{R}^{n}$ by means of the transformation

$$
\operatorname{Exp} t=\left(e^{t_{1}}, \ldots, e^{t_{n}}\right), \quad t=\left(t_{1}, \ldots, t_{n}\right) \in \mathbb{R}^{n} .
$$

Indeed, the pull-back function

$$
g(t):=\left(\mathbf{E x p}^{*} \omega\right)(t)=\omega\left(D_{1}, \bar{D}_{0} ; \mathbf{E x p} t\right)
$$


is convex on the convex set $G_{1}=\left\{t \in \mathbb{R}^{n}: \operatorname{Exp} t \in D_{1}\right\}$ and identically zero on $G_{0}=\left\{t \in \mathbb{R}^{n}: \operatorname{Exp} t \in \bar{D}_{0}\right\}$.

Then the complex Monge-Ampère operator $\left(d d^{c}\right)^{n}$ is transformed into the real Monge-Ampère operator $\mathcal{M A}$ defined for smooth convex functions $v$ as

$$
\mathcal{M A}[v](t)=\operatorname{det}\left(\frac{\partial^{2} v(t)}{\partial t_{j} \partial t_{k}}\right)
$$

and extended as a positive measure to all convex functions in $\mathbb{R}^{n}$ (see [13]). So, for any bounded plurisubharmonic function $u$ in $D_{1}$ which depends only on $\left|z_{1}\right|, \ldots,\left|z_{n}\right|$ and for any multicircular Borel set $A \subset \subset D_{1}$ we have

$$
\int_{A}\left(d d^{c} u\right)^{n}=(2 \pi)^{n} n ! \mathcal{M} \mathcal{A}\left[\operatorname{Exp}^{*} u\right](\widetilde{A})
$$

with $\widetilde{A}=\left\{t \in \mathbb{R}^{n}: \mathbf{E x p} t \in A\right\}$, since $\left(d d^{c} u\right)^{n}$ cannot charge the pluripolar set $A \cap\left\{z: z_{1} \ldots z_{n}=0\right\}$ (see details in [12]). Moreover, by [13], for any convex function $v$ in a domain $G_{1}$ and any measurable set $B \subset G_{1}$, we have

$$
\mathcal{M A}[v](B)=\operatorname{Vol} \gamma(B, v)
$$

where

$$
\gamma(B, v)=\bigcup_{t^{0} \in B}\left\{b \in \mathbb{R}^{n}: v(t) \geq v\left(t^{0}\right)+\sum_{k} b_{k}\left(t_{k}-t_{k}^{0}\right) \forall t \in G_{1}\right\}
$$

is the gradient image of the set $B$ for the surface $\left\{y=v(x): x \in G_{1}\right\}$.

Let $F:=\left\{t \in \mathbb{R}^{n}: \operatorname{Exp} t \in \partial D_{0}\right\}$. The Monge-Ampère measure $\left(d d^{c} \omega\right)^{n}$ is supported by $\partial D_{0}$, so, by (16) and (17), we have

$$
\int_{D_{1}}\left(d d^{c} \omega\right)^{n}=\int_{\partial D_{0}}\left(d d^{c} \omega\right)^{n}=(2 \pi)^{n} n ! \mathcal{M} \mathcal{A}[g](F) .
$$

Thus, due to (6), (18),

$$
\tau\left(\bar{D}_{0}, D_{1}\right)=n ! \operatorname{Vol} \gamma(F, g) .
$$

Note that since $g=0$ on $F$,

$$
\gamma(F, g)=\bigcup_{t^{0} \in F}\left\{b \in \mathbb{R}^{n}: g(t) \geq \sum_{k} b_{k}\left(t_{k}-t_{k}^{0}\right) \forall t \in G_{1}\right\} .
$$

Lemma 6. The relation $\gamma(F, g)=\Theta$ holds with $\Theta$ defined in (15).

Proof. Let $b \in \gamma(F, g)$. Then there is a point $t^{0} \in F$ such that $g(t) \geq$ $\sum_{k} b_{k}\left(t_{k}-t_{k}^{0}\right)$ for all $t \in G_{1}$. In particular, $\sum_{k} b_{k}\left(t_{k}-t_{k}^{0}\right) \leq 0$ for all $t \in G_{0}$ and so $b \in \mathbb{R}_{+}^{n}$.

For each $b \in \gamma(F, g)$ we have

$$
h_{D_{1}}(b)-h_{D_{0}}(b) \leq \sup _{t \in G_{1}} \sum_{k} b_{k}\left(t_{k}-t_{k}^{0}\right) \leq 1
$$

and thus $\gamma(F, g) \subset \Theta$. 
Conversely, let $b \in \Theta$. Then $h_{D_{0}}(b)=\sum_{k} b_{k} t_{k}^{0}$ for some $t^{0} \in F$. Take any $t \in G_{1}$. If $\sum_{k} b_{k}\left(t_{k}-t_{k}^{0}\right) \leq 0$, then certainly $\sum_{k} b_{k}\left(t_{k}-t_{k}^{0}\right) \leq g(t)$. On the other hand if $\sum_{k} b_{k}\left(t_{k}-t_{k}^{0}\right)>0$, then, taking into account (8),

$$
\begin{aligned}
\sum_{k} b_{k}\left(t_{k}-t_{k}^{0}\right) & \leq \frac{\sum_{k} b_{k}\left(t_{k}-t_{k}^{0}\right)}{h_{D_{1}}(b)-h_{D_{0}}(b)}=\frac{\sum_{k} b_{k} t_{k}-h_{D_{0}}(b)}{h_{D_{1}}(b)-h_{D_{0}}(b)} \\
& \leq \sup _{a \in \mathbb{R}_{+}^{n}} \frac{\sum_{k} a_{k} t_{k}-h_{D_{0}}(a)}{h_{D_{1}}(a)-h_{D_{0}}(a)}=g(t),
\end{aligned}
$$

and so $b \in \gamma(F, g)$, which completes the proof.

Lemma 6 together with (19) implies

Theorem 7. $\tau\left(\bar{D}_{0}, D_{1}\right)=n ! \operatorname{Vol} \Theta$.

Comparing this fact with Theorem 3 , we get Theorem 5 immediately.

\section{References}

[1] A. Aytuna, Stein spaces $M$ for which $\mathcal{O}(M)$ is isomorphic to a power series space, in: Advances in the Theory of Fréchet Spaces, T. Terzioğlu (ed.), Kluwer, Dordrecht, 1988, 115-154.

[2] A. Aytuna and T. Terzioğlu, Some applications of a decomposition method, in: Progress in Functional Analysis, K. D. Bierstedt et al. (eds.), Elsevier, 1992, 85-95.

[3] K. I. Babenko, On entropy of a class of analytic functions, Nauchn. Dokl. Vyssh. Shkoly Ser. Fiz.-Mat. 2 (1958), 9-13 (in Russian).

[4] E. Bedford and B. A. Taylor, A new capacity for plurisubharmonic functions, Acta Math. 149 (1982), 1-40.

[5] V. D. Erokhin, On asymptotic properties of $\varepsilon$-entropy of analytic functions, Dokl. Akad. Nauk SSSR 120 (1958), 949-952.

[6] S. D. Fisher and C. A. Micchelli, The n-widths of sets of analytic functions, Duke Math. J. 47 (1980), 789-801.

[7] W. K. Hayman and P. B. Kennedy, Subharmonic Functions, I, Academic Press, New York, 1976.

[8] A. N. Kolmogorov and V. M. Tikhomirov, $\varepsilon$-entropy and $\varepsilon$-capacity of sets in function spaces, Uspekhi Mat. Nauk 14 (1959), no. 2, 3-86 (in Russian).

[9] A. L. Levin and V. M. Tikhomirov, On Erokhin's theorem, Soviet Math. Surveys 23 (1968), 119-132.

[10] B. S. Mityagin, Approximative dimension and bases in nuclear spaces, Russian Math. Surveys 16 (1961), 59-127.

[11] T. V. Nguyen, Bases de Schauder dans certains espaces de fonctions holomorphes, Ann. Inst. Fourier (Grenoble) 22 (1972), no. 2, 169-253.

[12] A. Rashkovskii, Newton numbers and residual measures of plurisubharmonic functions, Ann. Polon. Math. 75 (2000), 213-231.

[13] J. Rauch and B. A. Taylor, The Dirichlet problem for the multidimensional MongeAmpère equation, Rocky Mountain J. Math. 7 (1977), 345-364.

[14] N. I. Skiba and V. P. Zahariuta, Estimates of n-diameters of some classes of analytic functions on Riemann surfaces, Math. Notes 19 (1976), 525-532. 
[15] N. I. Skiba and V. P. Zahariuta, Asymptotics of Kolmogorov diameters for classes of harmonic functions on spheroids, J. Approx. Theory 102 (2000), 175-188.

[16] H. Widom, Rational approximation and n-dimensional diameter, ibid. 5 (1972), 343-361.

[17] V. P. Zahariuta, On extendible bases in spaces of analytic functions of one and several variables, Siberian Math. J. 8 (1967), 204-216.

[18] - Extremal plurisubharmonic functions, Hilbert scales, and the isomorphism of spaces of analytic functions of several variables, I, II, Teor. Funktsiu Funktsional. Anal. i Prilozhen. 19 (1974), 133-157; 21 (1974), 65-83 (in Russian).

[19] —, Spaces of analytic functions and complex potential theory, Linear Topol. Spaces Complex Anal. 1 (1994), 74-146.

Department of Mathematics

Middle East Technical University

Ankara, Turkey

E-mail: aytuna@metu.edu.tr

Rostov State University

Rostov-na-Donu, Russia

and

Department of Mathematics

Middle East Technical University

Ankara, Turkey

E-mail: zaha@math.metu.edu.tr
Institute for Low Temperature Physics Kharkov, Ukraine E-mail: rashkovs@ilt.kharkov.ua 